\title{
Heterogeneity of antigenic constellation in human hepatocellular carcinoma
}

\author{
MIHAI CEAUSU $^{1 *}$, BOGDAN SOCEA $^{2,3}$, DRAGOS SERBAN $^{2,4^{*}}$, \\ CĂTĂLIN GABRIEL SMARANDACHE ${ }^{2,4}$, DRAGOŞ PREDESCU ${ }^{2,5}$, NICOLAE BACALBAŞA $^{6,7}$, \\ IULIAN SLAVU ${ }^{8}$, ADRIAN TULIN $^{8,9}$, LUCIAN ALECU $^{2,8}$ and ZENAIDA CEAUŞU ${ }^{10^{*}}$
}

\begin{abstract}
Departments of ${ }^{1}$ Pathology and ${ }^{2}$ Surgery, 'Carol Davila' University of Medicine and Pharmacy, 020021 Bucharest; ${ }^{3}$ Department of Surgery, 'Sf. Pantelimon' Emergency Hospital, 021659 Bucharest; ${ }^{4}$ IVth Department of Surgery, University Emergency Hospital, 050098 Bucharest; ${ }^{5}$ Department of Surgery, 'Sf. Maria' Hospital, 011172 Bucharest; ${ }^{6}$ Department of Obstetrics and Gynecology, 'Carol Davila' University of Medicine and Pharmacy, 020021 Bucharest;

${ }^{7}$ Department of Obstetrics and Gynecology, 'Dr. I. Cantacuzino' Clinical Hospital, 030167 Bucharest;

${ }^{8}$ Department of Surgery, 'Prof. Dr. Agrippa Ionescu' Clinical Emergency Hospital, 011356 Bucharest;

${ }^{9}$ Department of Anatomy, 'Carol Davila' University of Medicine and Pharmacy, 020021 Bucharest;

${ }^{10}$ Pathology Department, 'Sf. Pantelimon’ Emergency Hospital, 021659 Bucharest, Romania
\end{abstract}

Received October 23, 2020; Accepted November 24, 2020

DOI: $10.3892 / \mathrm{etm} .2021 .9701$

\begin{abstract}
Hepatocellular carcinoma is one of the primary liver malignancies responsible for over a million deaths per year worldwide (approximately 10\% of all deaths in the adult age range). The diagnosis of $\mathrm{HCC}$ can be difficult and often requires the use of more than one microscopic technique. A retrospective study was performed on a study batch of 42 cases that died of HCC due to metastasis or other secondary complications. Tissue samples were taken in order to investigate the tumour antigenic constellation by means of IHC method using a large variety of antibodies. In situ hybridization was also performed for albumin mRNA to assess the albumin expression in some selected cases. Telomerase activity was investigated using IHC method for the hTERT catalytic subunit. A cocktail of hepatic cytokeratins $(\mathrm{CK} 8,18)$ combined with Hep Par-1 and associated to albumin proved to be more powerful than albumin alone in differentiating HCC and increased the value of tumour diagnosis. hTERT expression was proportionally reverse to the tumour degree of differentiation, but was independent from the expression of tumour-proliferating indexes. The heterogeneity of the antigenic constellation in hepatocellular
\end{abstract}

Correspondence to: Dr Dragos Serban, Department of Surgery, 'Carol Davila' University of Medicine and Pharmacy, 37 Dionisie Lupu Street, 020021 Bucharest, Romania

E-mail: dragos.serban@umfcd.ro

${ }^{*}$ Contributed equally

Key words: hepatocellular carcinoma, histopathologic variability, immunophenotypical heterogeneity, molecular pathology, antigenic mosaicism carcinoma suggests an antigenic mosaicism, which can be expressed a synchronous or metachronous manner, depending on the tumour degree of differentiation.

\section{Introduction}

Among all primary liver cancers, hepatocellular carcinoma (HCC) accounts for $90 \%$ of cases, being the fourth leading cause of cancer death worldwide in 2018, with 841,000 new cases and 782,000 deaths annually (1).

Diagnosis of HCC is not always easy on simple hematoxylin and eosin (H\&E) stain. The diagnostic problems arise when a tumor shows pseudoglandular, trabecular, pleomorphic or clear cell differentiation (2-4).

The histological diagnosis poses many challenges particularly when dealing with liver biopsy specimens due to the heterogeneity of $\mathrm{HCC}$ and the difficulty to confirm hepatocellular differentiation in some instances (5).

The pleomorphism of cancer cells in HCC is caused by instability and disorganization of the cytoskeleton system, with an abnormal modulation of the intermediate filaments (such as cytokeratins, particularly CK8 and CK18), as well as with a deficiency of a cytoskeleton cross-linking protein. An unstable cytoskeleton may play a role in tumor transformation and progression, local invasion and distant metastasis (6).

Well-differentiated HCC may be differentiated from large regenerative or low-grade dysplastic nodules and high-grade dysplastic nodules by IHC features. A variety of antibodies were proposed for positive and differential diagnosis, each of them with its own limitation: $\alpha$-fetoprotein (AFP), Hep Par-1, Glypican (GPC-3), CK8, CK18, CK7, CK19, MOC-31, CD34, p-CEA, HSP70, glutamin synthetase, or albumin (7).

On the background of constantly accumulating data regarding human $\mathrm{HCC}$, the aim of the study was to gain further understanding of HCC diagnosis and behaviour. 


\section{Materials and methods}

Case selection for human tissue specimens. An extensive study was conducted on a batch of 42 cases with HCC, selected from a group of 72 patients with hepatocellular lesions (hepatitis B and C, cirrhosis and hepatic adenomas with or without dysplasia and malignant hepatic tumours, especially hepatocarcinomas).

The study batch comprised 36 men and 6 women (sex ratio 6:1), with age ranging from 10 to 77 years $(\mathrm{m}, 59$, $\mathrm{SD}, \pm 13.2$ ), in order to assess the tumour antigenic constellation in HCC by means of IHC, along with microscopic analysis of peritumoral stromal elements [such as tumour-infiltrating lymphocytes (TIL)] and the association between tumour and stroma.

The study was performed according to the World Medical Association Declaration of Helsinki and the tissue specimens were collected according to national legislation.

Tissue sampling and stains. Tissue samples from surgically resected specimens of $\mathrm{HCC}$ were taken for microscopic investigation. The selected tissue samples were fixed in $10 \%$ neutral-buffered formalin ( $\mathrm{pH}$ 7.0) for 24-48 $\mathrm{h}$ and paraffin embedded. Sections were cut at $5 \mu \mathrm{m}$ and stained with standard H\&E.

Additional special stains such as PAS, Gomori silver stain and van Gieson were carried out. Tissue samples were divided into appropriate-sized (3-5 $\mu \mathrm{m})$ sections for conventional microscopy and immunohistochemistry.

Immunohistochemical analysis (IHC) was performed for a vast panel of 13 antibodies, using sections displayed on slides treated first with poly-L-lysine. The panel comprised the following antibodies: CK8 (clone: B22.1, ready to use (RTU), Cell Marque), CK18 (clone: B23.1, RTU, Cell Marque), CK7 (clone: OV-TL 12/30, RTU, Cell Marque), CK19 (clone: A53-B/A2.26, RTU, Cell Marque), Hep Par-1 (clone: OCH1E5, RTU, Cell Marque), CD34 (clone: QBend, RTU, Cell Marque), CD68 (clone: KP-1, RTU, Cell Marque), Ki-67 (clone: MIB-1, RTU, Cell Marque), PCNA (clone: PC10, 1:200, Dako), $\alpha$-fetoprotein (poly, RTU, Cell Marque), pre-albumin (PAB, poly, 1:75, Dako), albumin (ALB, poly, 1:5,000, Dako) and telomerase (clone: NCL-hTERT, 1:30, Novocastra). IHC was performed on $3 \mu \mathrm{m}$ sections from formalin-fixed paraffin-embedded specimens.

An indirect tristadial Avidin-Biotin-Complex technique was used together with a NovoLink Polymer detection system which utilizes a novel control polymerization technology to prepare polymeric HRP-linker antibody conjugates, according to the manufacturer's specifications (Novocastra). Antigen retrieval technique (enzymatic pre-treatment) was performed as per the manufacturer specifications.

Molecular biology investigation was performed using a chromogenic in situ hybridization technique for hepatic albumin mRNA, using an oligonucleotidic cDNA probe with 51 base pairs, complementary to mRNA sequence which encodes human albumin.

Digital images obtained with an incorporated software program were processed and analysed with Microsoft Office Picture Manager (Washington, DC), running under Windows 10.
Statistical analysis. Statistical analysis was carried out using SPSS version 20 (IBM Corp.). The Student's t-test was used to determine the median, and mean \pm standard deviation as well as association between various parameters (monoclonal antibodies). $\mathrm{P}<0.05$ was considered statistically significant.

\section{Results}

The studied HCC occurred more frequently on cirrhotic liver, all with an advanced degree (grade II and III Edmondson in $62.5 \%$ of cases), with a trabecular-type predominance and the tumour cells were hepatocyte-like and pleomorphic types.

According to the recent WHO classification (8), 97\% of tumours were HCC-NOS (with a microscopic morphology of trabecular type in $69 \%$ of cases, pseudo-glandular and compact types, in 15 and $13 \%$ of cases, respectively) and $3 \%$ of tumours were HCC of scirrhous type, with marked desmoplastic reaction (Fig. 1). No fibro-lamellar type was observed.

A part of hepatic tumours, especially the well-differentiated types and those with clear cells kept the capacity of glycogen synthesis, emphasized by PAS stain; $>50 \%$ of HCC presented a peri-acinar reticulin network. Bile (with intra- or extra-cellular deposition) was also observed in $25 \%$ of cases.

CK8 was positive in $54.54 \%$ of cases, while CK18 was positive in $75.75 \%$ of cases. CK8 and 18 were better expressed by well-differentiated HCC than low-differentiated HCC. CK18 appeared to be more specific than CK8 for tumour hepatic tissue (Fig. 2).

Alpha-fetoprotein was expressed in $84.84 \%$ of cases, while Hep Par-1 was positive in $75.75 \%$ of cases (with a tendency of variation depending upon the degree of differentiation, but retaining its capacity of staining the tumour cells even in low-differentiated types). Hep Par-1 was diffusely expressed in the cytoplasm of tumour cells, with a focal or diffuse granular pattern (Fig. 2).

Well-differentiated tumours had a strong reaction to CD34 (81.81\% of cases), with a sinusoidal pattern (Fig. 2). Low-differentiated tumours with compact pattern had a weak reaction to $\mathrm{CD} 34$ with random pattern or were negative. Micro vascular density (MVD) was high in well-differentiated HCC with trabecular pattern and in low-differentiated HCC with pleomorphic cells; also, the increasing of MVD was accompanied by the Kupffer cells hyperplasia in HCC.

The density of intra-tumoral Kupffer cells infiltrate (assessed by CD68) was influenced directly by the density of peritumoral Kupffer cells infiltrate. ITO cell hyperplasia was independent of Kupffer cell hyperplasia and was accompanied by a dense infiltration with tumour histiocytes, others than Kupfer cells.

The density of tumour-infiltrating lymphocytes (TIL) was independent from the density of macrophages. Kupffer cell hyperplasia from the histiocytic infiltrate seemed to be more important in anti-tumour immune defence than TIL, the histiocytes coordinating as antigen presenting cells or the dynamic of the local anti-tumour immune response. Peritumoral TIL density did not influence the intra-tumoral TIL density.

The immune reaction to albumin showed positivity in trabecular type of $\mathrm{HCC}(56 \%)$ and was also positive in pleomorphic cytology types (43\%). Well-differentiated HCC expressed albumin better than low-differentiated tumours (Fig. 3). There was a strong direct relationship between the IHC expression of ALB and PAB in HCC. 


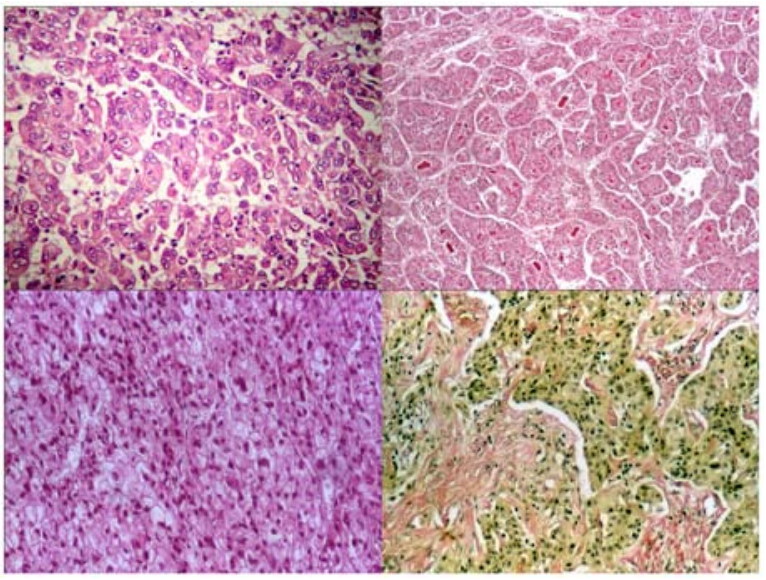

Figure 1. Various types of hepatocellular carcinoma: Trabecular (upper left panel: H\&E, x100), pseudoglandular (upper right panel: H\&E, x100), compact (lower left panel: H\&E, x100), scirrhous (lower right panel: Van Gieson staining, x100).

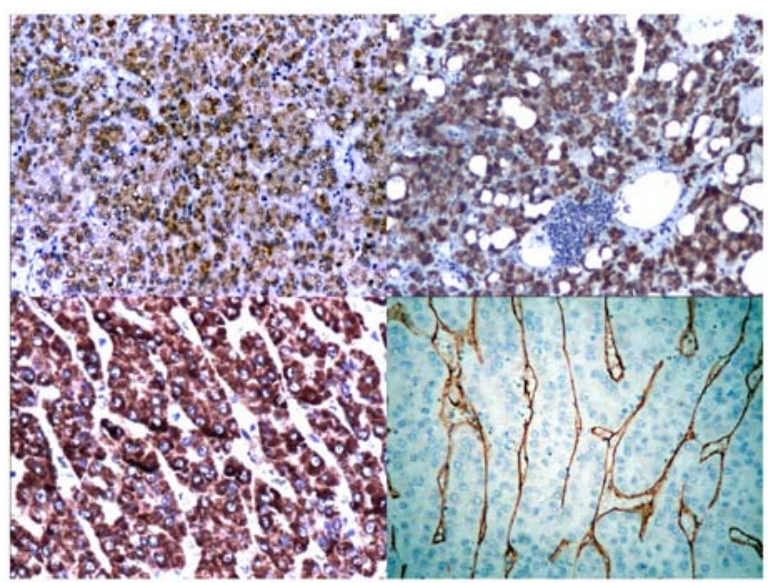

Figure 2. Immunophenotypical expression in hepatocellular carcinoma: CK8 (upper left panel: x100), CK18 (upper right panel: x100), Hep Par-1 (lower left panel: x100), CD34 (lower right panel: x100).

The variability of ISH reaction in studied HCC was high, recording extreme values (Fig. 3). There was also a slight reverse proportion between the intensity of $\mathrm{IHC}$ reaction and the intensity of ISH signal of ALB in HCC ( $\mathrm{r}=-0.3, \mathrm{P}=0.03)$.

IHC expression of PAB was independent of ISH expression of albumin mRNA, but there was a statistical correlation between PCNA and ISH for albumin $(r=0.5)$; the decreasing IHC expression of albumin was accompanied by an increasing of CK8 expression.

A cocktail of hepatic cytokeratins (CK8 and CK18) combined with Hep Par-1 and associated to albumin proved to be more powerful than albumin alone in differentiating HCC and increased the value of tumour diagnosis.

The catalytic subunit of the telomerase (hTERT) showed a homogenous nuclear reaction or in clusters in all types of HCC (Fig. 4).

hTERT expression was reverse proportional to the tumour degree of differentiation (low-differentiated tumours had a high level of expression and vice versa). Interestingly, the hTERT expression was independent from the expression of tumoral proliferating indexes (Ki-67 or PCNA).

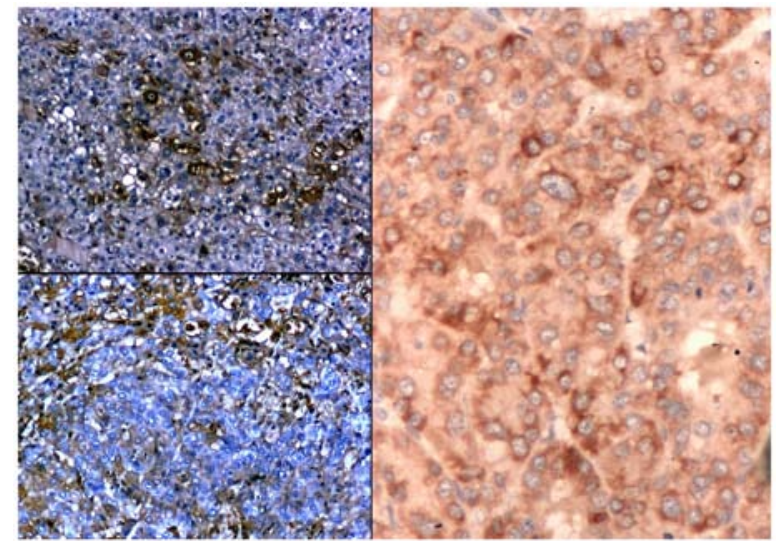

Figure 3. Albumin expression in hepatocellular carcinoma: Pre-albumin (upper left panel: IHC, x100), albumin (upper right panel: IHC, x100), in situ hybridization for albumin mRNA (right panel: x200).

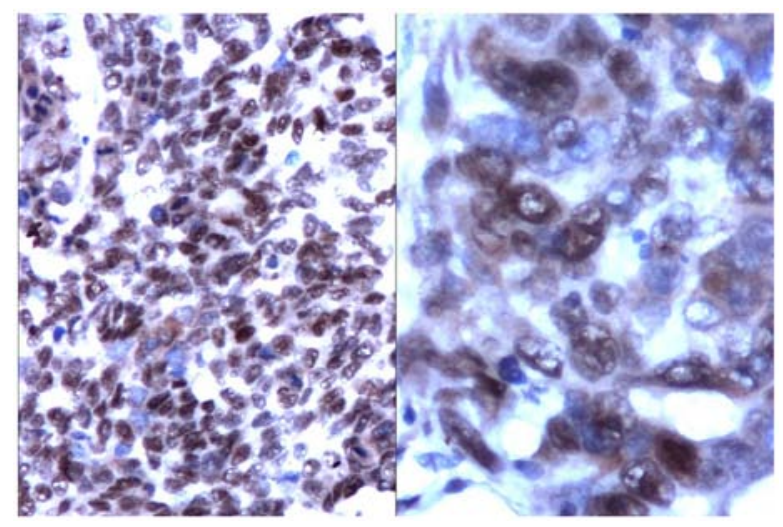

Figure 4. hTERT expression in tumour cell nuclei in HCC (left panel: IHC, x200); detail with nuclear clusters (right panel: IHC, x400)

There was a statistically significant association between CD34 and hTERT ( $\mathrm{r}=-0.4, \mathrm{P}=0.0002)$, which suggests a reverse proportion between telomerase activity and microvascular density.

\section{Discussion}

Trabecular and pseudo-glandular types are well-differentiated forms of HCC that must be distinguished from cirrhosis with atypia, atypical adenomatous hyperplasia and high-grade dysplastic nodule, which only by morphological means, makes the differential diagnosis very difficult. The identification of stromal invasion can be a clue for HCC, in contrast to the other aforementioned lesions, which do not invade the surrounding tissues.

Adenomatous hyperplasia (originally coined by Edmondson) is divided into 'ordinary' (common) or atypical types. The first one does not have a neoplastic nature (representing a macro-regenerative nodule in cirrhosis), while the second one is a pre-neoplastic lesion, with various degrees of dysplasia, based on cytological and architectural changes (9).

There is a considerable overlap in microscopic features in well-differentiated hepatocellular carcinoma and other hepatic non-neoplastic lesions (such as the distinction of well-differentiated HCC and hepatic adenoma in 
non-cirrhotic liver or distinguishing between early HCC from high-grade dysplastic nodule, in a cirrhotic liver), requiring the use of immunohistochemistry and other techniques for diagnosis (10-12).

Normal and neoplastic hepatocytes express CK8 and CK18 and are generally negative for CK7, CK19, and CK20 (13). However, in our study, normal peritumoral hepatocytes did not express CK7 and 19, but tumour hepatocytes expressed these types of cytokeratin in $25 \%$ of cases, sometimes simultaneously with CK8 and 18. This may suggest the possibility of immunophenotype change of tumour hepatocytes during the progression of HCC.

Therefore, the cytokeratin set $(\mathrm{CK} 8,18,7,19)$ along with Hep Par-1 expressed by HCC in our studied cases showed the possibility of the existence of an antigenic mosaic, which can be expressed as synchronous or metachronous, depending on tumour differentiation degree.

In an exhaustive study on 799 patients with a large panel of antibodies ( $\alpha$-fetoprotein, CD34, CK7, CK19, glypican-3, $\mathrm{Ki}-67$, glutamine synthetase and $\beta$-catenin), it was found that immunohistochemical expression of these markers in HCC in a non-cirrhotic and cirrhotic liver was comparable, having limited additional value to characterise HCC in non-cirrhotic livers. Additionally, none of the immunohistochemical stains were associated with a worse overall survival (14).

Hep Par 1 is a monoclonal antibody that was developed using formalin-fixed tissue from failed allograft liver, which turned to be a sensitive and specific marker for HCC (15).

In a recent comparative cross-sectional study, Hep Par-1 proved useful in differentiating hepatocellular carcinoma from metastatic carcinoma, taking histopathology as a gold standard (16).

According to a study conducted by Kakar et al (17), Hep Par 1 and polyclonal carcinoembryonic antigen are the most reliable markers for hepatocellular differentiation, but they have low sensitivity for poorly differentiated cases, requiring additional markers such as glypican-3 or different types of cytokeratin.

Glypican-3 (GPC-3) is a membrane-anchored proteoglycan and was designated as an oncofetal protein, which was normally expressed in fetal liver, but not in normal adult liver. Certain studies showed that GPC-3 was expressed in $\sim 72 \%$ of HCCs, but not in normal liver or hepatic adenoma (18).

GPC-3 seems to be a relatively sensitive and specific marker in the positive diagnosis of $\mathrm{HCC}$, and when it is coupled with other markers such as CD34, CD10 and AFP is useful in differentiating HCC from dysplastic nodules, cirrhotic regenerative nodules, focal nodular hyperplasia and hepatocellular adenoma (19).

In combination with a complete CD34 immunostaining pattern, GPC-3 greatly improves the accuracy of distinguishing between malignant hepatic lesions and benign lesions (20). As HCC is a highly vascularized tumour, angiogenesis plays a fundamental role in progression of hepatocellular carcinoma $(21,22)$. In a relative recent study, microvascular density determined by CD34 and CD105 (endoglin) expression proved to be useful as an additional parameter to distinguish between benign and malignant hepatic nodules, underlining that CD34 had higher average microvascular density scores than CD105 in HCC, with a more uniform positivity pattern (23).
Alpha-fetoprotein (AFP) is an oncofetal protein; its expression in a tumor is relatively specific for hepatocellular differentiation, if germ cell tumors can be excluded. The IHC sensitivity is about $40 \%$, but serum AFP levels are helpful in the diagnosis of HCC and surveilling response to therapy for HCC (24).

A clinic-pathological analysis of 375 cases revealed that besides tumour size, tumour differentiation and vessel invasion (as important factors which affect the prognosis of patients with HCC), Hep Par-1 and AFP have predictive significance in HCC, along with GPC-3 and CD34 (25).

In patients with serum negative alpha-fetoprotein, hepatocellular carcinoma with focal nodular hyperplasia showed high CD34 and CK19, and low PCNA level (26).

Albumin in situ hybridization (ISH) is specific for hepatocellular differentiation and has a high sensitivity $(\sim 90 \%)$. Controversial findings however have been reported $(27,28)$.

In one study, even if ISH for albumin mRNA was expressed in all HCCs from the study batch, it was also positive in intrahepatic cholangiocarcinoma and focally positive in gallbladder adenocarcinoma and a subset of other neoplasms, which could be a potential pitfall (29).

On the other hand, in another study, mRNA albumin ISH showed high correlation with Hep Par 1 immunoreactivity; their combined use for diagnosis of HCC had a sensitivity of $100 \%$ in this population (30).

Overall, branched chain ISH performed on manual and automated mode is a sturdy assay for detecting albumin with reliable sensitivity for poorly differentiated HCCs. When interpreted in combination with Hep Par-1 and Arginase-1, ISH for mRNA albumin offers a high level of sensitivity and specificity (31).

Human telomerase has 3 elements: A template, an associated protein and a catalytic subunit (hTERT). Telomerase activity depends mainly on telomerase reverse transcriptase (hTERT) in hepatocellular carcinoma.

The correlation of the expressions of hTERT, c-myc and Ki-67 in HCC were closely associated, according to a study from 2009 (32), the overexpression of these three factors playing a vital role in the progress of HCC.

In our study, the hTERT expression was independent from the expression of tumoral proliferating indexes, such as Ki-67 or PCNA, but its expression was higher in low-differentiated tumours than in high-differentiated tumours.

In another study meant to assess the correlation between hTERT and PTEN expression in HCC, it was found that PTEN and hTERT have different roles in the development of HCC (33). Thus, authors of that study showed that a significantly negative correlation between PTEN and hTERT gene expression indicates that hTERT activation and upregulation may be conferred by the loss of PTEN gene expression in HCC. The combined detection of PTEN and hTERT, however, may provide critical clinical evidence for the diagnosis and biological behaviour of HCC (33).

In conclusion, the heterogeneity of the antigenic constellation in hepatocellular carcinoma suggests an antigenic mosaicism, which can be expressed as synchronous or metachronous, depending on the tumor degree of differentiation, and a targeted use of a cytokeratin 'cocktail' (CK8 and CK18, CK7 and CK19), combined with Hep Par-1, Glypican and 
CD34, along with albumin detection (by both means of IHC and ISH) increases the value of the positive and differential diagnosis of HCC.

\section{Acknowledgements}

Not applicable.

\section{Funding}

Not applicable.

\section{Availability of data and materials}

The datasets used and/or analyzed during the current study are available from the corresponding author on reasonable request.

\section{Authors' contributions}

MC and ZC performed the histological examinations and IHC, designed the study and had major contributions in writing the manuscript. BS, DS, CGS, DP and NB analyzed and interpreted the patient data. DS, IS, AT and LA searched the literature for similar work and articles, analyzed the data and had major contributtions in writing the manuscript. All authors read and approved the final manuscript.

\section{Ethics approval and consent to participate}

The study was conducted according to the World Medical Association Declaration of Helsinki, using a protocol approved by the local Bioethics Committee from 'St. Pantelimon' Emergency Clinical Hospital (Bucharest, Romania). All patients have previously signed an informed written consent about hospitalization, treatment and a possible future publication of data.

\section{Patient consent for publication}

Not applicable.

\section{Competing interests}

The authors declare no conflict or competing interests.

\section{References}

1. Jiang Y, Han QJ and Zhang J: Hepatocellular carcinoma: Mechanisms of progression and immunotherapy. World $\mathrm{J}$ Gastroenterol 25: 3151-3167, 2019.

2. Amarapurkar AD, Rege JD, Joshi AS, Vaiphei $\mathrm{K}$ and Amarapurkar DN: Utilization of antihepatocyte clone OCH1E5 (Hep Par 1) in histological evaluation of liver tumors. Indian $\mathbf{J}$ Pathol Microbiol 49: 341-344, 2006.

3. Negrut N, Khan SA, Bungau S, Zaha DC, Aron CR, Bratu O, Diaconu CC and Ionita-Radu F: Diagnostic challenges in gastrointestinal infections. Rom J Mil Med 123: 83-90 2020.

4. Draghici T, Negreanu L, Bratu OG, Stoian AP, Socea B, Neagu TP, Stanescu AM, Manuc D and Diaconu CC: Paraneoplastic syndromes in digestive tumors: A review. Rom Biotechnol Lett 24: 813-819, 2019.

5. Quaglia A: Hepatocellular carcinoma: A review of diagnostic challenges for the pathologist. J Hepatocell Carcinoma 5: 99-108, 2018.
6. Lai YS, Cheng CC, Lee MT, Chao WT, Lai YC, Hsu YH and Liu YH: The prognostic value of cytokeratin and sal-like protein 4 expression in hepatocellular carcinoma and intra-hepatic cholangiocarcinoma in Taiwan. Int J Med Sci 15: 1746-1756, 2018.

7. Ferrell LD: Hepatocellular carcinoma and its variants. In: Odze and Goldblum Surgical Pathology of the GI Tract, Liver, Biliary Tract, and Pancreas. 3rd edition. Elsevier Saunders Publishing House, pp1549-1557, 2015.

8. Torbenson MS, Ng IOL, Park YN, Roncalli M and Sakamoto M: Hepatocellular carcinoma, chapter 8: Tumours of tumours of the liver and intrahepatic bile ducts. In: WHO Classification of Tumours, Digestive System. 5th edition, pp229-239, 2019.

9. Kutlesic C, Katic V, Veliekovic L and Katic K: Atypical adenomatous hyperplasia in liver cirrhosis. Arch Oncol 12 (Suppl 1): S10-S11, 2004

10. Shafizadeh N and Kakar S: Diagnosis of well-differentiated hepatocellular lesions: role of immunohistochemistry and other ancillary techniques. Adv Anat Pathol 18: 438-445, 2011.

11. Epîngeac ME, Găman MA, Diaconu CC, Gad M, and Găman AM: The evaluation of oxidative stress in obesity. Rev Chim (Bucharest) 70: 2241-2244, 2019.

12. Dumitru N, Cocolos A, Caragheorgheopol A, Dumitrache C, Bratu OG, Neagu TP, Diaconu CC and Ghemigian A: Collagen-the ultrastructural element of the bone matrix. Rev Chim (Bucharest) 69: 1706-1709, 2018.

13. Van Eyken P, Sciot R, Paterson A, Callea F, Kew MC and Desmet VJ: Cytokeratin expression in hepatocellular carcinoma: An immunohistochemical study. Hum Pathol 19: 562-568, 1988.

14. Witjes CD, ten Kate FJ, Verhoef C, de Man RA and Jzermans JN: Immunohistochemical characteristics of hepatocellular carcinoma in non-cirrhotic livers. J Clin Pathol 66: 687-691, 2013.

15. Fan Z, van de Rijn M, Montgomery K and Rouse RV: Hep Par 1 antibody stain for the differential diagnosis of hepatocellular carcinoma: 676 tumors tested using tissue microarrays and conventional tissue sections. Mod Pathol 16: 137-144, 2003.

16. Hanif R and Mansoor S: Hep par-1: A novel immunohistochemical marker for differentiating hepatocellular carcinoma from metastatic carcinoma. J Coll Physicians Surg Pak 24: 186-189, 2014.

17. Kakar S, Gown AM, Goodman ZD and Ferrell LD: Best practices in diagnostic immunohistochemistry: Hepatocellular carcinoma versus metastatic neoplasms. Arch Pathol Lab Med 131: 1648-1654, 2007

18. Wang XY, Degos F, Dubois S, Tessiore S, Allegretta M, Guttmann RD, Jothy S, Belghiti J, Bedossa P and Paradis V: Glypican-3 expression in hepatocellular tumors: Diagnostic value for preneoplastic lesions and hepatocellular carcinomas. Hum Pathol 37: 1435-1441, 2006.

19. DU JL, Wei LX and Wang YL: Expression and clinicopathologic significance of GPC3 and other antibodies in well-differentiated hepatocellular carcinoma. Zhonghua Bing Li Xue Za Zhi 40: 11-16, 2011 (In Chinese).

20. Coston WM, Loera S, Lau SK, Ishizawa S, Jiang Z, Wu CL, Yen Y, Weiss LM and Chu PG: Distinction of hepatocellular carcinoma from benign hepatic mimickers using Glypican-3 and CD34 immunohistochemistry. Am J Surg Pathol 32: 433-444, 2008.

21. Suceveanu AI, Stoian AP, Mazilu L, Voinea F, Hainarosie R, Diaconu CC, Pituru S, Nitipir C, Badiu DC, Ceausu I and Suceveanu AP: Interferon-free therapy is not a trigger for hepatocellular carcinoma in patients with chronic infection with hepatitis C virus. Farmacia 66: 904-908, 2018.

22. Gheorghe G, Pantea Stoian A, Gaman MA, Socea B, Neagu TP, Stanescu AMA, Bratu OG, Mischianu DLD, Suceveanu AI, and Diaconu CC: The benefits and risks of antioxidant treatment in liver diseases. Rev Chim (Bucharest) 70: 651-655, 2019.

23. Segatelli V, de Oliveira EC, Boin IF, Ataide EC and Escanhoela CA: Evaluation and comparison of microvessel density using the markers CD34 and CD105 in regenerative nodules, dysplastic nodules and hepatocellular carcinoma. Hepatol Int 8: 260-265, 2014.

24. Lau SK, Prakash S, Geller SA and Alsabeh R: Comparative immunohistochemical profile of hepatocellular carcinoma, cholangiocarcinoma, and metastatic adenocarcinoma. Hum Pathol 33: 1175-1181, 2002.

25. Du JL, Wang YL, Shi HY, Guo AT and Wei LX: Expression of glypican-3, hepatocyte antigen, alpha-fetoprotein, CD34 and CD10 in hepatocellular carcinoma: A clinicopathologic analysis of 375 cases. Zhonghua Bing Li Xue Za Zhi 41: 309-13, 2012 (In Chinese) 
26. Cui DJ, Wu Y and Wen DH: CD34, PCNA and CK19 expressions in AFP-hepatocellular carcinoma. Eur Rev Med Pharmacol Sci 22: 5200-5205, 2018

27. Surcel M, Huică RI, Munteanu AN, Isvoranu G, Pîrvu IR Ciotaru D, Constantin C, Bratu O, Căruntu C, Neagu M and Ursaciuc C: Phenotypic changes of lymphocyte populations in psoriasiform dermatitis animal model. Exp Ther Med 17: $1030-1038,2019$

28. Plotogea O, Ilie M, Sandru V, Chiotoroiu A, Bratu O and Diaconu C: Cardiovascular and metabolic consequences of liver transplantation: A review. Medicina (Kaunas) 55: E489, 2019.

29. Nasir A, Lehrke HD, Mounajjed T, Said S, Zhang L, Yasir S, Shah SS, Chandan VS, Smyrk TC, Moreira RK, et al: Albumin in situ hybridization can be positive in adenocarcinomas and other tumors from diverse sites. Am J Clin Pathol 152: 190-199, 2019.
30. Kakar S, Muir T, Murphy LM, Lloyd RV and Burgart LJ: Immunoreactivity of Hep Par 1 in hepatic and extrahepatic tumours and its correlation with albumin in situ hybridization in hepatocellular carcinoma. Am J Clin Pathol 119: 361-366, 2003.

31. Shahid M, Mubeen A, Tse J, Kakar S, Bateman AC, Borger D, Rivera MN, Ting DT and Deshpande V: Branched chain in situ hybridization for albumin as a marker of hepatocellular differentiation: Evaluation of manual and automated in situ hybridization platforms. Am J Surg Pathol 39: 25-34, 2015.

32. Fang YW: Expression and significance of hTERT, c-myc and Ki-67 in hepatocellular carcinoma. Xi Bao Yu Fen Zi Mian Yi Xue Za Zhi 25: 338-340, 2009 (In Chinese).

33. Zhou X, Zhu H and Lu J: PTEN and hTERT gene expression and the correlation with human hepatocellular carcinoma. Pathol Res Pract 211: 316-319, 2015. 